\title{
Mathematical Modeling of HIV Dynamics After Antiretroviral Therapy Initiation: A Review
}

\author{
Pablo S. Rivadeneira, ${ }^{1,2}$ Claude H. Moog, ${ }^{2}$ Guy-Bart Stan, ${ }^{3}$ Cecile Brunet, ${ }^{4,5}$ François Raffi,, ${ }^{4,5}$ \\ Virginie Ferré, ${ }^{4,5}$ Vicente Costanza, Marie J. Mhawej, ${ }^{2}$ Federico Biafore, ${ }^{6}$ Djomangan A. Ouattara, ${ }^{2}$ \\ Damien Ernst, ${ }^{7}$ Raphael Fonteneau, ${ }^{7}$ and Xiaohua Xia ${ }^{8}$
}

\begin{abstract}
This review shows the potential ground-breaking impact that mathematical tools may have in the analysis and the understanding of the HIV dynamics. In the first part, early diagnosis of immunological failure is inferred from the estimation of certain parameters of a mathematical model of the HIV infection dynamics. This method is supported by clinical research results from an original clinical trial: data just after 1 month following therapy initiation are used to carry out the model identification. The diagnosis is shown to be consistent with results from monitoring of the patients after 6 months. In the second part of this review, prospective research results are given for the design of individual anti-HIV treatments optimizing the recovery of the immune system and minimizing side effects. In this respect, two methods are discussed. The first one combines HIV population dynamics with pharmacokinetics and pharmacodynamics models to generate drug treatments using impulsive control systems. The second one is based on optimal control theory and uses a recently published differential equation to model the side effects produced by highly active antiretroviral therapy therapies. The main advantage of these revisited methods is that the drug treatment is computed directly in amounts of drugs, which is easier to interpret by physicians and patients.
\end{abstract}

Key words: aid for diagnosis; AIDS; apoptosis; dynamical systems; HIV; modeling; nonlinear control; pharmacodynamics; pharmacokinetics

\section{Introduction}

$\mathbf{M}$ ATHEMATICAL MODELING HAS MADE a substantial impact on our thinking and understanding of HIV-1 infection. A large number of deterministic models have been developed to describe the immune system and its interaction with HIV as well as the effects of drug therapy. The extended cooperation between A. Perelson and D. Ho using clinical data and mathematical modeling has been used to elucidate the principles that govern HIV and immune system dynamics in relation to antiviral drug therapy. ${ }^{1-3}$

Other studies revealed the lifetime of infected cells, which actively produce copies of the virus, and the high replication rate of HIV in the body. These results reinforce the idea that HIV is a dynamic disease encompassing a number of different time scales, running from hours to months.

In this review, the HIV dynamics are analyzed from the point of view of control systems. Clinical research results show that parameter identification methods constitute an efficient means to characterize critical patients, just 1 month after initiation of a new therapy. ${ }^{4-7}$ Thus, parameter identification methods provide information to physicians that can be very useful for the diagnosis of patients under clinical failure. ${ }^{8}$ Prospective research of control methods recently published (without clinical data) is discussed as well and research directions are given for the design of an optimal,

\footnotetext{
${ }^{1}$ Nonlinear System Group, INTEC-Facultad de Ingeniería Química (UNL-CONICET), Santa Fe, Argentina.

${ }^{2}$ L'UNAM, IRCCyN, UMR-CNRS 6597, Nantes, France.

${ }^{3}$ Imperial College London, Department of Bioengineering, South Kensington Campus, United Kingdom.

${ }^{4}$ Infectious Diseases, University Hospital, Nantes, France.

${ }^{5}$ EA4271 Immunovirologie et polymorphisme génétique, Nantes University, Nantes, France.

${ }^{6}$ Center of Applied Mathematics, School of Science and Technology, National University of San Martin, San Martín, Buenos Aires, Argentina.

${ }^{7}$ University of Liège, Department of Electrical Engineering and Computer Science, Montefiore Institute, Liège, Belgium.

${ }^{8}$ Department of Electrical, Electronic and Computer Engineering, University of Pretoria, Pretoria, South Africa.
} 
nonconstant drug dosage therapy to control the virus dynamics and to minimize undesired side effects. ${ }^{9-11}$

Following this line of interdisciplinary research, the achievements that this review article emphasizes are as follows:

1. A clinical trial has been set up for the first time to show that mathematical systems analysis is useful to help clinicians in an early diagnosis and in taking decisions for treating HIV-infected patients,

2. An optimal (time-varying) dosage is generated by taking into account the desired dynamics for the immune system as well as the side effects generated by antiretroviral drugs after initiation of the treatment. This is one of the perspectives offered by a mathematical approach.

3. The control action (therapy) is computed in terms of the drug dosage in milligrams, which is significant for real-life practitioners, whereas earlier articles consider the efficiency of drugs as the control input. Pharmacokinetic and pharmacodynamic models are included in the HIV model.

The outline of this review is as follows. The next section introduces a model of the infection dynamics. The clinical research results are then described, including the methods, and a discussion of the results. Namely, the identified model is argued to be an efficient tool for characterizing critical patients and for the diagnosis of clinical failure within 1 month after initiation of a new therapy. More prospective research is reviewed, and strategies for the control of the infection dynamics using drug treatments are revisited in the framework of engineering and computational methods. For these prospective strategies, only in silico results are presented because no clinical study is available yet. Finally, conclusions are presented.

\section{Basic Modeling of the Infection Dynamics}

Several nonlinear models have been developed to describe the dynamics of the HIV-1 virus, which take into account the kinetics of the HIV infection with different cell populations; for example, macrophages, cytotoxic T lymphocytes, latently infected $\mathrm{CD} 4^{+} \mathrm{T}$ cells as well as the lymphoid compartments. $^{12,13}$

With a goal of providing useful diagnostic information and controlling the infection, the main characteristics of the HIV dynamics can be modeled based on a relatively simple set of ordinary differential equations. In fact, the simplest model of the HIV/AIDS dynamics (which is used herein) can be understood through the interactions of healthy $\mathrm{CD}^{+}$cells $(T)$, infected $\mathrm{CD} 4^{+}$cells $\left(T^{*}\right)$, and free viruses $(V)^{3}$ :

$$
\begin{gathered}
\dot{T}=s-\delta T-\left(1-\eta_{1}\right) \beta T V-A T T^{*} \\
\dot{T}=\left(1-\eta_{1}\right) \beta T V-\mu T^{*} \\
\dot{V}=\left(1-\eta_{2}\right) k T^{*}-c V
\end{gathered}
$$

where healthy $\mathrm{CD}^{+}{ }^{+}$cells $(T)$ are produced from the thymus at a constant rate $s$ and die with a half-life time equal to $\frac{1}{\delta}$ The healthy cells are infected by the virus at a rate that is proportional to the product of their population size and the amount of free virus particles. The proportionality constant $\beta$ is an indication of the effectiveness of the infection process. The term $A T T^{*}$ is included to take into account that, in the presence of infected cells $\left(T^{*}\right)$, healthy $\mathrm{CD} 4^{+}$cells undergo activation-induced apoptosis. ${ }^{7,14}$

The infected $\mathrm{CD}^{+}$cells result from the infection of healthy CD4 cells and die at a rate $\mu$. Free virus particles are produced from infected CD4 cells at a rate $k$ and die with a half-life time equal to $\frac{1}{c}$. The parameters $\eta_{1}$ and $\eta_{2}$ represent the efficacy of an anti-HIV treatment, which is generally a cocktail of drugs composed of reverse transcriptase inhibitors and protease inhibitors. Reverse transcriptase inhibitors are usually considered to affect the parameter $\beta$ and protease inhibitors, the parameter $k .^{3,15,16}$ In particular, $\eta_{1}=\eta_{2}=0$ represents a null therapy, while $\eta_{1}=\eta_{2}=1$ denotes a $100 \%$ effective therapy. Equation 3 takes into account the fact that one virus particle can only infect one single $\mathrm{CD} 4{ }^{+}$cell.

\section{Clinical Research: EDV05 Clinical Trial Design and Early Diagnosis of Therapeutic Failure}

Among a general population of HIV-infected patients starting a new therapy, some individuals will eventually undergo a so-called immunological failure. One main contribution reviewed here is to show that parameter identification is able to predict such failures after 1 month from the beginning of the treatment, while the immunological failure usually takes 6 months to be diagnosed during a standard clinical monitoring.

As previously stated, immunological failure is clinically declared when the amount of CD4 $\mathrm{T}$ cells remains under $200 \mathrm{CD} 4 / \mathrm{mm}^{3}$ during 6 months of efficient treatment. $5,17,18$ A treatment is defined as efficient, if it yields a decrease of the viral load below the detectable threshold of 50 copies/ $\mathrm{mL}$ and keeps the viral load below this limit afterwards.

A central paradox for HIV patients who are in immunological failure is that their viral load seems too low to be able to deplete the $\mathrm{CD} 4{ }^{+}$population by direct killing alone. In this respect, the first studies ${ }^{8}$ relate immunological failure to a dysfunctional thymus, which is unable to produce a sufficient amount of healthy CD4 ${ }^{+} \mathrm{T}$ cells. However, a different point of view is argued in the section Prospective Research: Infection Control and Drug Dosage, which relates immunological failure to an important activation-induced apoptosis phenomenon in the transient stage of the infection. This point of view is supported by several research articles, ${ }^{7,14,19-24}$ based on thymic function having little chance to vary within a healthy adult population. Whether immunological failure is predominantly due to a badly damaged thymus or to an important activation-induced apoptosis phenomenon remains an open question.

\section{Methods}

To generate useful data for the characterization of the immunological failures, the EDV05 trial was initiated at the University Hospital of Nantes in February 2005, and six patients were included in the first part of this trial. In 2007 and 2008, 10 other patients were enrolled. The main conditions for each patient (female or male) to be included in the trial were as follows: aged over 18 years old, having been infected by the HIV-1 or HIV-2 type virus, having no co-infection 
with hepatitis $\mathrm{B}$ or hepatitis $\mathrm{C}$ virus during the 6 months preceding their inclusion in the trial, starting an antiretroviral treatment at the beginning of the trial, and not showing any resistance to the treatment that will be administrated.

The measured data consisted of the viral load and the total CD4 T cells. Eleven samples were collected during 3 months, but the relevant information about the dynamics (six or seven blood samples) was taken within the first 20 days. This specific sampling protocol enables a first estimation of all the parameters of the mathematical model (Equations 1-3 with $\left.\eta_{1}=0\right){ }^{4,8}$ From day 30 to day 90, only four or five other samples were taken, with a sampling time of about 15 days. Since the measured viral load values depend on the laboratory protocol used to quantify the RNA copies, ${ }^{16,25-28}$ it was decided for each patient to perform all the measurements of the viral load at the same time with the same protocol. Thus, the 11 blood samples from each patient were kept frozen $\left(\right.$ at $\left.-80^{\circ} \mathrm{C}\right)$ until the end of the trial (see Fig. 1). The measurements were done at the same time using the Roche Taqman $48^{\mathrm{TM}}$ test, with a detectable threshold of 50 copies/mL (CD4 T cells and virus load values for the 16 patients are given in table 2 of Prud'homme et al. ${ }^{29}$ ).

Based on clinical observations, Mhawej et al. ${ }^{4}$ concluded that the identification of the parameters of the dynamical model (Equations $1-3$ with $\eta_{1}=0$ ) allows defining a quantifiable immunological status. Since available measurements typically contain the total (uninfected and infected) CD4 T cells and free virions, the parameters of the proposed model can only be determined from these output measurements (blood samples) under certain identifiability conditions. ${ }^{30}$

The identification method used in this reviewed study was developed by Ouattara ${ }^{5}$ and Ouattara et al..$^{8}$ and implemented in software available in reference 6 . The method is based on the Monte Carlo approach, combined with a simplex optimization method. To avoid local optima, random initial conditions were drawn from uniform distributions in the admissible parameter space and corresponding estimates were computed. Using the median and interquartile range of the estimates' distributions, reliable estimations and confidence intervals of the parameters were deduced. For Equations 1-

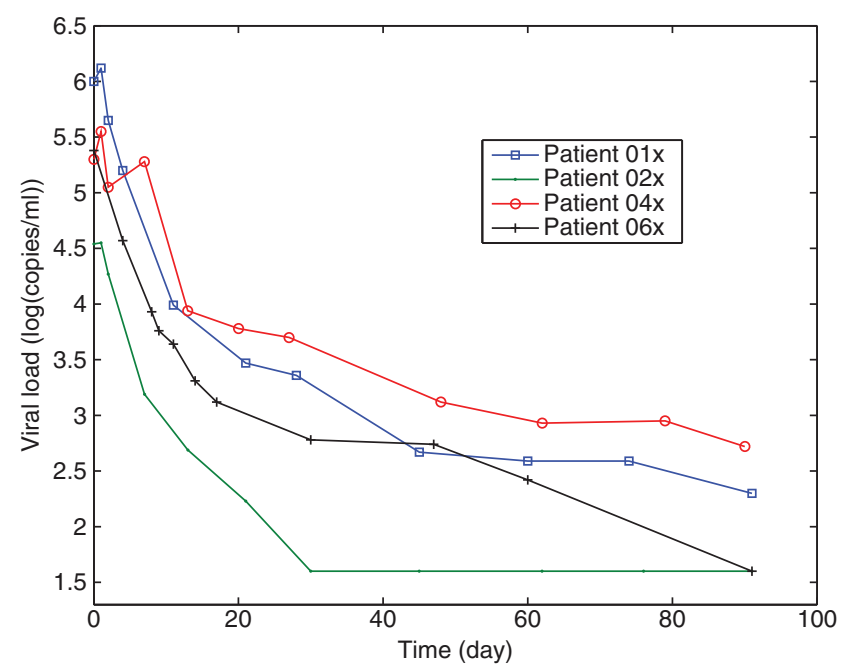

FIG. 1. Viral load time evolution for four patients. The samples are taken frequently at the beginning of the trial.
3 (with $\eta_{1}=0$ ), a number of 1000 randomizations was sufficient to obtain stable and robust results. ${ }^{8}$ The calibration of the algorithm was as follows:

- Life-time parameters were set to constant values, which relied on typical values published in Ouattara et al. ${ }^{8}$ These were $\delta=0.01 / \mathrm{d}, \mu=0.05 / \mathrm{d}$, and $c=0.3 / \mathrm{d}$.

- According to the biological characteristics of the infection, the other parameters were constrained to be bounded in the following intervals: $s \in[1 E-5,20], \beta \in[1 E-$ 20,1], $A \in[1 E-20,1], k \in[1 E-20,1 E+8]$.

- The uniform distributions $U$ used for the initial conditions were: $\quad U_{A} \in[1 E-10,0.5], U_{\beta} \in[1 E-10,1 E-5]$, $U_{s} \in[1 E-5,10], U_{k} \in[1,5 E+3]$.

Parameters in the mathematical model of the HIV/AIDS infection are related to the immunological status of the patient. For instance, $s$ is the natural production rate of CD4 $\mathrm{T}$ cells by the thymus. So, a low value for $s$ is representative of a badly damaged immune system. Similarly, a large value for $A$ indicates an important activation-induced apoptosis phenomenon.

When the apoptosis phenomenon is not considered, an auxiliary mathematical characterization of the immunological failure can be stated using the identified parameters as follows.

The immune potential is given by the maximal level of $\mathrm{CD}^{+} \mathrm{T}$ cells that can be reached if a $100 \%$ efficient treatment is administered. This maximal level is obtained by setting $V=0$ in Equation 1 and computing its equilibrium point as $\Delta_{\max }=\frac{\mathrm{s}}{\delta}$. So, patients with a normal function of the thymus must have a $\Delta_{\max }>200 \mathrm{CD} 4 / \mathrm{mm}^{3}$.

But in practice, it is desirable to reach the equilibrium $\Delta_{\max }$ within a reasonable time (around 6 months in general). Thus, a more restrictive evaluation of the status of the immune system is provided by the time $t_{200}$ required to reach the critical threshold of $200 \mathrm{CD} 4 / \mathrm{mm}^{3}$. For patients who are not in immunological failure, $t_{200}$ is less than 6 months. More generally, $t_{\Delta}$ denotes the time required to reach the level $\Delta$ of CD4 T cells count in the case of a $100 \%$ efficacy of the therapy. $t_{\Delta}$ is computed as follows ${ }^{17}$ :

$$
t_{\Delta}=\max \left\{\frac{1}{\delta}\left[-\log _{e}\left(1-\frac{\delta}{s} \Delta\right)+\log _{e}\left(1-\frac{\delta}{s} T(0)\right)\right], 0\right\}
$$

Summarizing, patients are declared to be critical if they belong to one of the following groups:

- A low value of the parameter $s(s \leq 3)$ and a high value of the parameter $A\left(A>10^{-2}\right)$

- $\Delta_{\max }>200 \mathrm{CD} 4 / \mathrm{mm}^{3}$

- $\Delta_{\max }>200 \mathrm{CD} 4 / \mathrm{mm}^{3}$, but $t_{200}>6$ months

\section{Results}

To characterize critical patients, two identifications of the parameters were done using the data collected in the first month of the trial; that is, the first seven or eight samples were used. The first identification only considered a degraded functionality of the thymus; that is, the parameter $A$ was taken equal to zero. The second identification took into account the activation-induced apoptosis phenomenon but a normal thymic function $\left(s=6 \mathrm{~mm}^{3} / \mathrm{d}\right)$. The numerical results of the identification of the parameters $\left.s\right|_{A=0}$ and 
$\left.A\right|_{s=6}$ for each patient in the EDV05 trial and the analysis of the remaining parameters of the dynamical model can be found in previous articles. ${ }^{4,8,29}$

\section{Discussion}

In Rivadeneira et al., ${ }^{29}$ the last column of table 1 displays the theoretical time $t_{200}$ necessary to recover a CD4 level above $200 \mathrm{CD} 4 / \mathrm{mm}^{3}$. This theoretical time $t_{200}$ was computed from Equation 4 using the parameter identification method based only on the samples from the first 3 weeks after initiation of the therapy. According to this theoretical time $t_{200}$, five patients are predicted to be in immunological failure since they will not recover the desired CD4 level of $200 \mathrm{CD} 4 / \mathrm{mm}^{3}$ within 6 months. The remaining 11 patients are predicted to recover within 6 months, and among those, 10 patients are even predicted to recover the desired CD4 level within 3 months.

Based on the identification of parameters $s$ or $A$ only, six patients are declared to be critical: they are exactly those for whom $t_{200}>3$ months. Patients 3, 7, 11, 13, 15, and 16 were critical patients because they had the highest values of apoptosis parameter $A$ and concurrently the lowest values of the parameter $s$. The immunological failure of patient 3 was clinically confirmed after 3 months of monitoring. Patient 11 was 59 years old at the time of the trial and was the oldest among the 16 patients who were included. In this special case, the small value of $s$ or the high value of $A$ rather indicated poor dynamics of the immune system, which is standard at the age of 60 . Similar observations were made for patient 13 who was 49 years old at the beginning of the trial. The clinical monitoring of patient 15 shows co-existing tuberculosis that weakened the immunological system and explained the estimated values of $s$ and $A$. Patient 16 was only 18 years old when he was enrolled in the trial. He had a very low level of $3 \mathrm{CD} 4 / \mathrm{mm}^{3}$. This patient was very young and his immunological system proved after 6 months its ability to recover from its critical status. ${ }^{8,29} t_{200}$ lay within the range 3 months $<t_{200}<6$ months (predicted from Equation 4 and can be found in table 1 in reference 29).

The last column of the table 2 in Rivadeneira et al. ${ }^{29}$ displays the immunological status of the patients after 6 months. The latter was fully consistent with the previous results. The 11 patients reaching the level $200 \mathrm{CD} 4 / \mathrm{mm}^{3}$ within 6 months were all correctly identified, which suggests a good sensitivity of the measure based on the identified parameters $s$ and $A$. The five patients having a level below $200 \mathrm{CD} 4 / \mathrm{mm}^{3}$ after 6 months were correctly identified as well, which again suggests a good specificity of the measure. These latter statements deserve an exhaustive statistical study, which is beyond the scope of this review article.

The conclusion of these results is that the identification of the parameters in Equations 1-3 can be used for the early diagnosis of immunological failure just after the first month following initiation of a new therapy. The peculiarities of each patient such as therapy adherence, age, or other circumstances must be taken into account by the physician to make a final clinical decision.

\section{Prospective Research: Infection Control and Drug Dosage}

In the previous section, the parameters of a model were identified for patients under treatment. In this section, a model for a patient without therapy will be considered and used to review two special drug-scheduling therapies designed using control engineering methods.

HIV drug-scheduling strategies have drawn attention from the biomedical and control engineering communities and many control strategies have been proposed for therapeutic treatment. For instance, state feedback and optimal control of HIV infection have been explored in several works. ${ }^{9,15,31-36}$ However, most of these methods consider the efficacy of the drugs as the control variable, which results in strategies that are quite hard to use in practice since drug efficacy is typically not expressed in terms of administered drug amounts. To circumvent this drawback, pharmacokinetic and pharmacodynamic considerations can be included in the model to relate efficiency with drug dosage, ${ }^{10}$ as it is shown next. Therapeutic treatments, designed using control theory, are theoretical results with potential important impact. The parameters used in the following models were extracted from data available in the HIV literature and correspond to an average patient not following any therapy.

Antiretroviral therapy slows the clinical progression of HIV infection at the beginning of the therapy. However drug resistance due to viral mutation typically occurs after some time and poses a challenging problem for long-term treatments. Some studies have speculated that alternating between drug regimens on a fixed schedule might forestall therapeutic failure. Early stages of the virus evolution are assumed to be mutation free. During further stages, the virus mutation can no longer be ignored. This is considered for instance in Hernandez-Vargas et al., ${ }^{37}$ in which the mutation of the virus is modeled using switching systems, which include 64 virus strains and three drug combinations. This issue remains the topic of ongoing research.

In the results included in this review, the mathematical models do not take into account drug resistance and mutation of the virus. The drug therapies generated based on these models were designed to act at the first stages of the therapeutic regimen, during which the probability that the virus develops some mutation is still low. Two control policies are considered: feedback linearization and optimal control.

\section{Feedback linearization control applied to HIV drug scheduling}

The problem of drug administration is classically divided into two phases: a so-called pharmacodynamic phase that relates the concentration of drugs at the site of action to the magnitude of the effect produced, modeled as $\eta=\frac{w(t)}{w(t)+w_{50}}$, and a pharmacokinetic phase that relates dose, frequency, and route of administration to drug level-time relationships in the body, mathematically described as $\dot{w}=-K w+$ $u(t) \delta\left(t-\tau_{k}\right)$. The parameter $w_{50}$ is the concentration of drug that lowers the viral load by $50 \%, K$ is the elimination rate constant of the drug, $u(t)$ is the amount of drug expressed in milligrams, and $\tau_{k}$ is the time at which the patient takes the drug.

Pharmacokinetic and pharmacodynamic drug parameters are usually evaluated in vitro, whereas in vivo parameters are needed. Pharmacological characteristics of any drug vary significantly when evaluated in vitro or in vivo. Thus, the in vivo parameters given by Legrand et al. ${ }^{38}$ are used herein. The model used is as follows: 


$$
\begin{gathered}
\dot{T}=s-\delta T-\beta T V \\
\dot{T}^{*}=\beta T V-\mu T^{*} \\
\dot{V}=\left(1-\frac{w}{w+w_{50}}\right) k T^{*}-c V \\
\dot{w}=-K w+u(t) \delta\left(1-\tau_{k}\right)
\end{gathered}
$$

The control strategy is computed taking into account that

i. The effect of antiretroviral drugs has a strong impact on the value of the parameter $k$ (therefore $\eta_{1}$ is assumed to be zero) as is explained in Mhawej et al. ${ }^{39}$

ii. A discrete drug trajectory $u(t)$ is derived by means of a nonlinear control strategy such as feedback linearization for impulsive control systems. ${ }^{11}$

Based on these aforementioned considerations, the amount of drug really present in the body can be computed. A full description of the technique is provided by Mhawej et al. ${ }^{10}$ and Rivadeneira et al. ${ }^{11}$

This setup based on impulsive control systems allows variations of the control variable in amounts of drug (milligrams) instead of in terms of their efficiency and describes in a realistic way how the patient follows the therapy.

Some results of this control method are illustrated in Fig. 2, which represents the infection evolution for different values of the dosing interval $\tau_{k}$. The simulation is performed when zidovudine (ZDV) is given twice a day ( $\tau_{k}=0.5$ days) following the closed-loop control strategy. In Fig. 2a, the virus load approaches the desired healthy equilibrium in the expected time, according to the requirement to reach 200 cells $/ \mathrm{mm}^{3}$ within 6 months. In Fig. 2b, a discrete time scheduling of the antiretroviral drug can be seen.

As we can see, the inclusion of pharmacokinetic and pharmacodynamic models using in vivo parameters along with impulsive control becomes a valuable tool to test and design therapeutical strategies. Note that this result is observed when considering a ZDV monotherapy. More significant results could be obtained with multitherapies. Discussions about the use of multitherapies or the convenience of administering the drug once, twice, or three times per day; that is, to consider drug impulses at variable times $\tau_{k}$ are left open for future works.

One major advantage of the control schemes cited (like state feedback) is their ability to minimize the viral load by means of a time-varying administration strategy aimed at optimally adjusting the efficiency of the drugs to the dynamics of the patient. This means that if the efficiency of the drug is related to its dosage, no full dosage is needed all the time. The benefits appear through a reduction of the side effects caused by the use of dynamically nonoptimal drug doses.

\section{Control of HIV infection with minimum side effects}

In Costanza et al., ${ }^{9}$ extended dynamics of the HIV infection model were developed. A new differential equation was included to model the generation of side effects due to medication. After that, optimal control theory was implemented to minimize side effects. This subsection resumes those main results. Side effects appear and evolve during
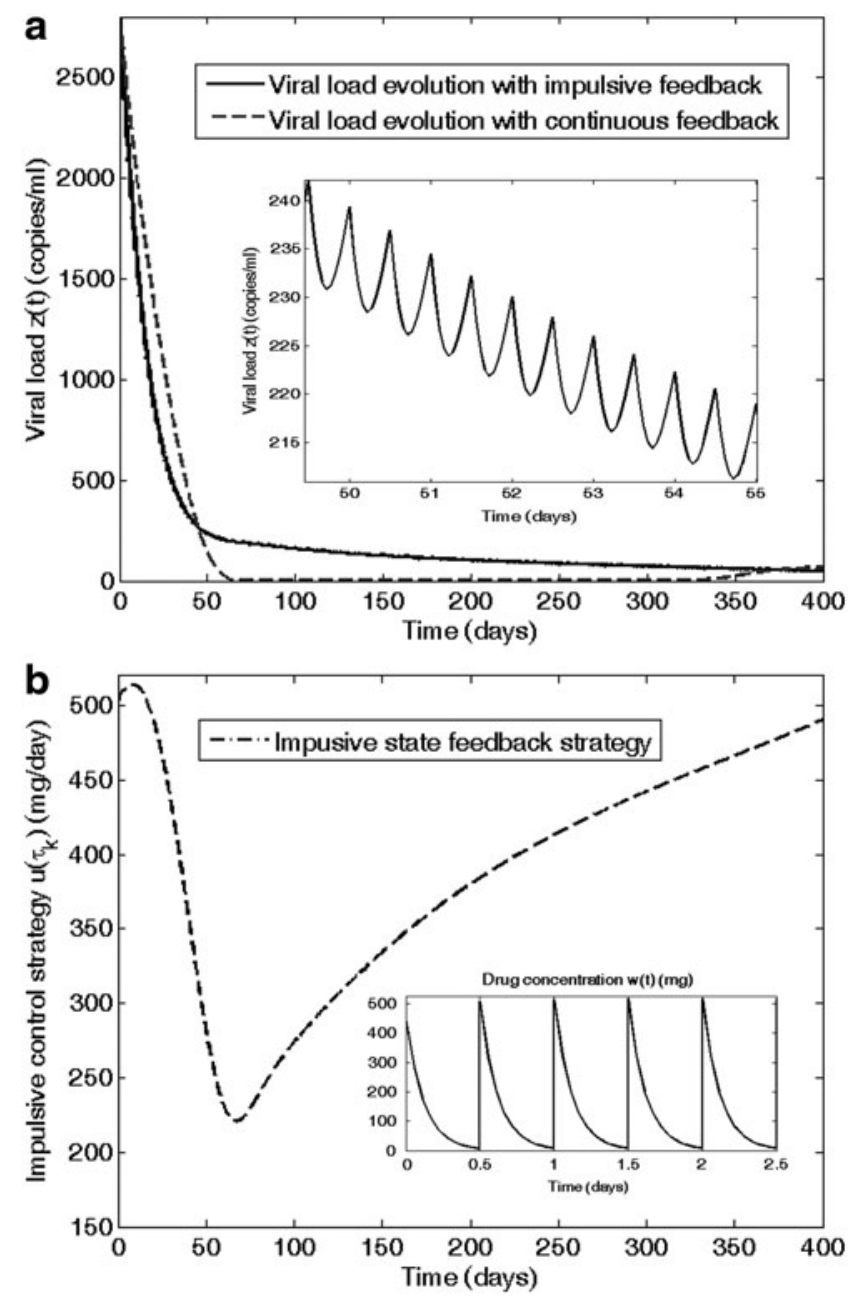

FIG. 2. (a) Time evolution of HIV infection during zidovudine (ZDV) monotherapy is depicted on the left side. (b) The doses, administrated twice a day, are computed by means of a feedback linearizing impulsive control; their amplitude is drawn on the right side.

the therapy process, frequently persisting even if medication is discontinued. Since the characteristics of these side effects have a wide range, it is virtually impossible to identify all disorders provoked by medication in a patient, and sometimes it is difficult to confirm that an eventually arising disease, different from HIV, is a side effect. However, a comparison study on efficacy and side effects for different doses of ZDV has shown that the incidence of some moderate to severe side effects was dose related. ${ }^{40,41}$

Using the percentages of patients affected by these side effects in the median follow-up period as a reference, an estimate of the probability that HIV patients present any confirmed side effect up to a given time after the beginning of medication can be computed. In the same line of reasoning, these data were used to describe (approximately) the dynamic behavior of such a probability $p(t)$ through an equation of the form ${ }^{9.32}$ :

$$
\dot{p}=\left(d_{0}+d_{1} u+d_{2} u^{2}+d_{3} u^{3}\right) p(1-p)\left(V+V_{h}\right)^{w}, p(0)=p_{0} .
$$

Several considerations based on the structure of Equation 9 need to be taken into consideration at this stage: 
- The variable $u$ denotes the drug dose, expressed in grams.

- The variable $p$, being a probability, should range in $[0,1]$. As far as it is recognized that side effects exist due to medication, it seems consistent that the probability must be nondecreasing while drugs consumption persists. Given the medical consensus recommending avoiding interruptions in the medication, the condition $u>0$ for significant periods of time seems unavoidable.

- The growth of $p$ as a function of the drug dose $u$ must be nonlinear. Let us assume, without loss of generality, that this nonlinearity accepts a power series description. This is just an approximation that should be improved with the incoming of new experimental data.

- A small probability of side effects is always present, which amounts to consider $p_{0} \neq 0$.

- Side effects are analyzed only in medicated HIVinfected patients; that is, the viral load is always nonzero $(V \neq 0)$. Consequences of the ingestion of drugs, which are part of highly active antiretroviral therapy, in HIV-free persons will not be considered. The value of the parameter $\eta$ must be fine-tuned with experimental data.

- The reason for adding a parameter $V_{h}$ is to avoid the persistence of side effects when the viral load is nearly zero but the patient is still consuming a high drug dose.

- The parameter $d_{0}$ should be small but positive because even when medication is interrupted ( $u=0$ for some time), side effects will probably increase due to previous drug history.

From the numerical data presented by the Nordic Medical Research Council, ${ }^{40}$ a set of parameter values have been fitted and tested by simulating new cases and analyzing the results. The final adopted values can be seen in Costanza et al. ${ }^{9}$ Empirical approximations for pharmacokinetic and pharmacodynamic equations were used to relate the real dose with its efficiency. Since only monotherapies of ZDV were considered, $\eta_{2}$ is assumed to be zero and $\beta=\beta_{0}-\beta_{1} u-\beta_{2} u^{2}$, where $0<u<1.6 \mathrm{~g}$. Costanza et al. ${ }^{9,32}$ provide further details. The resulting model is

$$
\begin{gathered}
\dot{T}=s-\delta T-\left(\beta_{0}-\beta_{1} u-\beta_{2} u^{2}\right) T V \\
\dot{T}^{*}=\left(\beta_{0}-\beta_{1} u-\beta_{2} u^{2}\right) T V-\mu T^{*} \\
\dot{V}=k T^{*}-c V \\
\dot{p}=\left(d_{0}+d_{1} u+d_{2} u^{2}+d_{3} u^{3}\right) p(1-p)\left(V+V_{h}\right)^{w}
\end{gathered}
$$

Based on Equations 10-13, a complete scheme of optimization was carried out to minimize the side effects and maximize the immune response, as it is briefly shown hereafter. These objectives can be represented mathematically by the following equation:

$$
\begin{aligned}
\min _{u} J(u)= & \min _{u} \int_{t_{0}}^{T}\left(a_{1} V(t)+a_{2}(T(t)-\bar{T})^{2}+a_{4} p(t)\right. \\
& \left.+a_{3} u^{2}(t)\right) d t+a_{5}(V(t)-\bar{V})^{2} d t
\end{aligned}
$$

where $t_{0}$ is the initial time, $T$ is the final time of optimization, and $\bar{T}$ and $\bar{V}$ are desired levels of $\mathrm{CD}^{+}{ }^{+} \mathrm{T}$ cells and virions, respectively. The initial conditions were assumed to simulate a patient with a recently discovered infection, for instance $(0)=850 \mathrm{CD} 4 / \mathrm{mm}^{3}, T *(0)=41$ cells $/ \mathrm{mm}^{3}, V(0)=$ 3760 copies $/ \mathrm{mL}, p(0)=0.05$.

The main goal $^{9}$ was to abate the infection, while generating as few side effects as possible. The optimal control strategy was recovered from the Dynamic Programming solution to the optimal control problem formulated in Equation 14. ${ }^{9}$ The corresponding optimal state trajectory was also stored during the solution process and is depicted in Figs. 3-5. At each sampling time there also starts a curve, a solution to the continuous-time flow of Equations 10-13, showing the expected evolution of the system during the next sampling period. That curve is truncated at the end of this period, according to the adopted discretization of the states. The evolution of the "protocol" trajectories (i.e., those corresponding to running the dynamics alone, with $u(t) \equiv 1.2$ and no optimization whatsoever) has been included in the figures for comparison.

The optimal solutions succeed in reducing the number of viral copies below 50 copies $/ \mathrm{mL}$ in the desired time of 180 days. The virion population decreases strongly at the beginning and the end of the treatment, remaining at intermediate levels during most of the period, which is not too bad as long as no deleterious "peaks" occur. At the extreme stages the optimal drug dose is high, but during most of the treatment the drug dose values stay around $0.6 \mathrm{~g}$, half of the tested protocol value. Also, during the application of the control strategy the number of $\mathrm{T}$ cells is always higher than $600 \mathrm{CD} 4 /$ $\mathrm{mm}^{3}$, which is considered clinically acceptable. ${ }^{42}$

The value of $a_{4}=0.08$ produced optimal trajectories whose partial costs (different terms in the total cost of Equation 14) had an incidence higher than expected on the total cost. The incidence of these individual costs does not show a strict monotone behavior with the variation of the weight $a_{4}$. However, in general terms it is noted that the growth of $a_{4}$ produces a less significant optimal $p(t)$ trajectory (Fig. $5)$, as expected. A smaller $p(t)$ is associated with a smaller

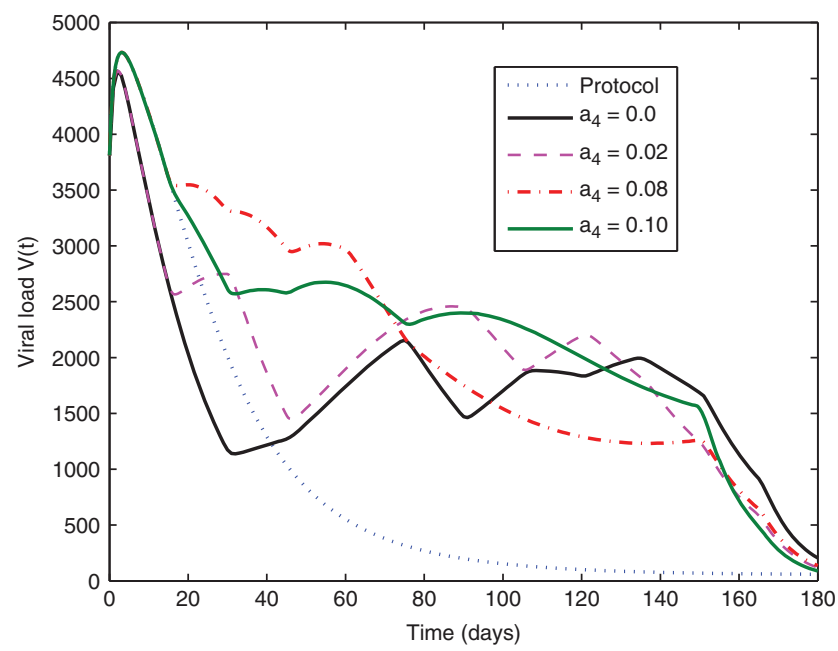

FIG. 3. Comparison between optimal $V$ virus trajectories for medications that take or do not take side effects into account, controlled by the weight parameter $a_{4}$ in the cost function $J(u)$. 


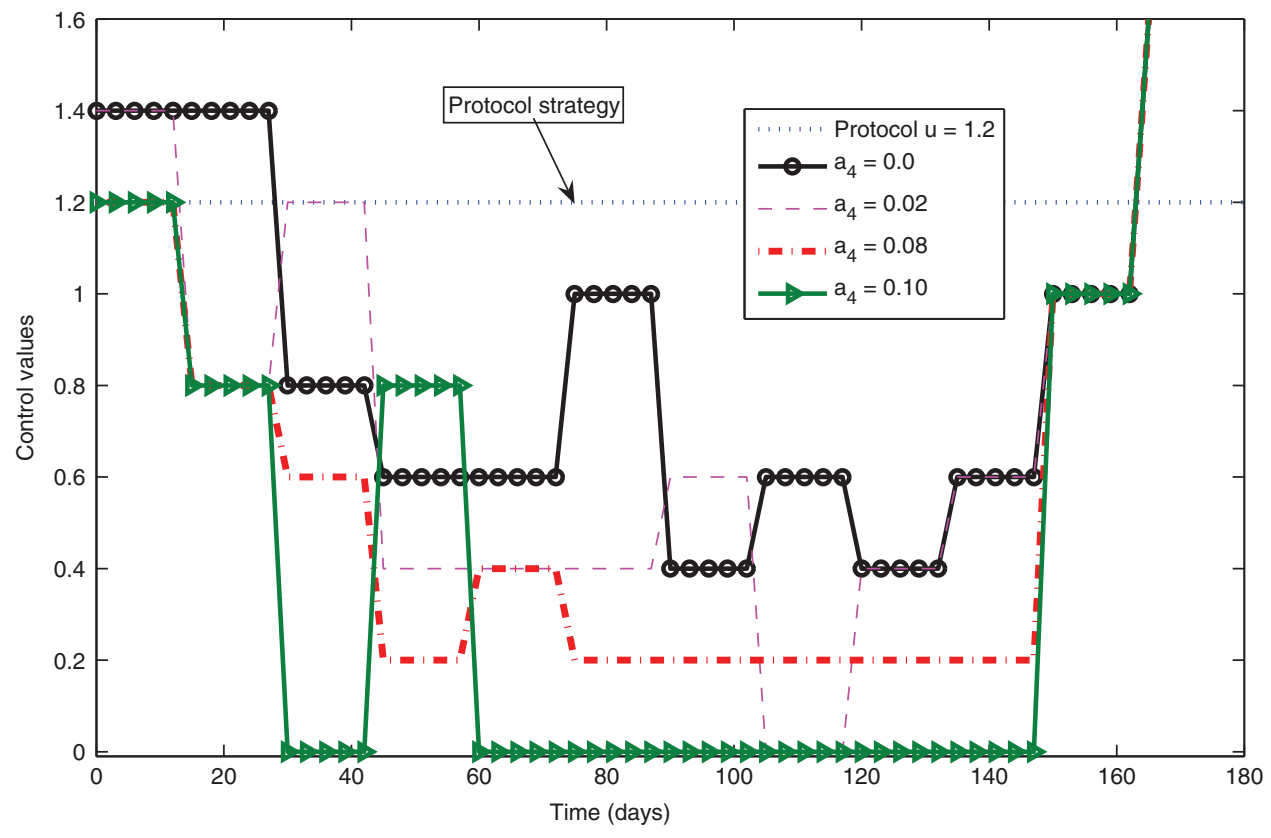

FIG. 4. Optimal control strategies for different decisions regarding the cost weight $a_{4}$. $u(t)$, and since $a_{3}$ was kept fixed, a decrease of the incidence of drug costs is found for increasing $a_{4}$, while, as a natural counterpart, the significance of penalties for departures from desired state values increases. Keeping the remaining cost weights fixed, the value of $a_{4}=0.1$ should be taken as an acceptable upper bound, since its corresponding optimal control trajectory shows two transient treatment interruptions (for $t \in\{[30,45] \cup[60,150]\}$ see Ouattara et al. ${ }^{8}$ for more details). The latter may not be desired as it may favor the mutation of the virus. This aspect is beyond of the scope of this review, and is left for further work that can be conducted considering switching systems for instance.

\section{Conclusions and Research Perspectives}

Mathematical models and systems and control solutions, as provided by feedback and optimal control theory, have

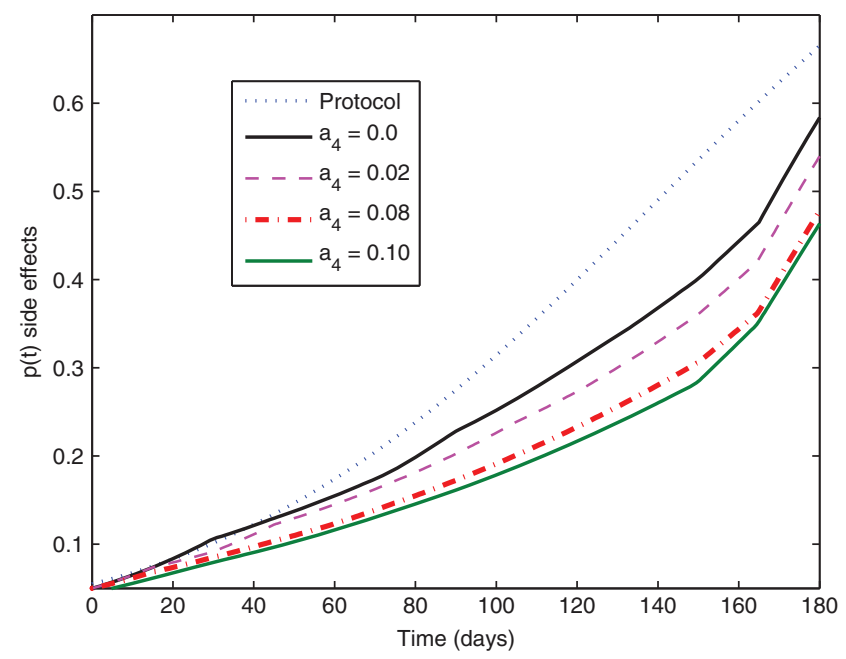

FIG. 5. Optimal evolution of side effects for different decisions regarding the cost weight $a_{4}$. proved to be very useful in the study of several biological and biochemical systems. Their use allows us to improve the prediction capabilities of existing HIV models and to substantiate variable drug dosages that minimize a cost function, while balancing combined objectives. Such interdisciplinary work requires knowledge of the results, tools, and languages coming from a disjointed scientific community. The results obtained until now provide a promising outlook at the outcomes and results that can be expected from a mathematical approach of HIV research: improvement of the knowledge of underlying dynamic processes behind the disease and development of new pharmacological strategies.

The obtained results show that engineering methods provide an alternative approach for analyzing the dynamics of the HIV infection process. For example, engineering methods provide a successful way to design clinical trials based not only on medical knowledge but also on quantitative analysis, allowing for a reduction in the number of blood samples required from each patient, and minimizing monetary cost.

Based on the presented models, mathematical analyses, and control methods, physicians have more tools at their disposal to help the decision-making process at different stages of the infection. The estimation of some parameters appearing in the presented mathematical models can be used in the diagnosis as was shown in the section Prospective Research: Infection Control and Drug Dosage. The pharmacodynamic and pharmacokinetic parameters that have been used in this review may be replaced by adequate parameters corresponding to more recent drug therapies whenever available. It is worth mentioning that the values of such parameters as the free virus decay rate, ${ }^{43}$ the death rate of actively infected cells, ${ }^{44}$ and the turnover rate of target cells ${ }^{45,46}$ are still controversial. A multidrug treatment yields also a more sophisticated model. ${ }^{47}$ The methods reviewed here can be adapted and used without major modification by changing the values of some parameters; their general conclusions remain valid.

Post diagnosis, the control schemes presented in this review article are useful to design optimal treatment strategies 
customized for each patient. The design of multi-objective costs can generate optimal doses according to the immunological and virological state of the patient as well as mitigating side effects and costs.

Research perspectives include a long-term drug optimization that takes into account the risk of treatment-resistant mutations, within the framework of so-called switching systems, since the characteristics of the virus dynamics depend on the virus strain, which can abruptly change once mutations occur. This investigation is mandatory for a real-life application and is the topic of future research.

\section{Acknowledgments}

This research work was first supported by the PROTEA financial program (Grant No. 05 STIC F4/SA) and the CNRS/ NRF (Grant No. 18385) in the frame of the joint France/ South-Africa research agreement. This work was partly supported by the Tournesol project No. 20424TF and by a CNRS-INRIA/MINCyT project. The authors wish to thank the University Hospital (CHU) of Nantes and the Hospital of La Roche-sur-Yon for having provided clinical data and supervised patients' recruitment in the clinical trial No. BRD 04/12-G sponsored by CHU of Nantes (called EDV05 in this article). Damien Ernst is a Research Associate of the Belgian FRS-FNRS, of which he acknowledges the financial support. Guy-Bart Stan acknowledges the support of the UK-based Centre for Synthetic Biology and Innovation (EPSRC grant EP/G036004/1).

\section{Author Disclosure Statement}

No competing financial interests exist.

\section{References}

1. Ho DD, Neumann AU, Perelson AS, et al. Rapid turnover of plasma virions and CD4 lymphocytes in HIV-1 infection. Nature. 1995;373:123-126.

2. Perelson AS, Essunger P, Cao Y, et al. Decay characteristics of HIV-1-infected compartments during combination therapy. Nature. 1997;387:188-191.

3. Perelson AS, Nelson PW. Mathematical analysis of HIV-1 dynamics in vivo. SIAM Review. 1999;41:3-44.

4. Mhawej MJ, Brunet-François C, Fonteneau R, et al. Apoptosis characterizes immunological failure of HIV infected patients. Control Engineering Practice. 2009;17:798-804.

5. Ouattara DA. Mathematical analysis of the HIV-1 infection: Parameter estimation, therapies effectiveness, and therapeutical failures. In 27th Annual International Conference of the IEEE Engineering in Medicine and Biology Society, Shanghai, China, September 2005.

6. Ouattara DA, Mhawej MJ, Moog CH. IRCCyN web software for the computation of HIV infection parameters 2007. Available at http://vih.irccyn.ec-nantes.fr

7. Stan GB, Belmudes F, Fonteneau R, et al. Modelling the influence of activation-induced apoptosis of $\mathrm{CD} 4+$ and CD8 + T-cells on the immune system response of a HIV infected patient. IET Syst Biol. 2008;2:94-102.

8. Ouattara DA, Mhawej MJ, Moog CH. Clinical tests of therapeutical failures based on mathematical modeling of the HIV infection. Joint special issue of IEEE Transactions on Circuits and Systems and IEEE Transactions on Auto- matic Control, Special issue on Systems Biology 2008;53: 230-241.

9. Costanza V, Rivadeneira PS, Biafore FL, et al. Taking into account side effects for HIV medication. IEEE Trans Biomed Eng. 2010;57:2079-2089.

10. Mhawej MJ, Moog CH, Biafore F, et al. Control of the HIV infection and drug dosage. Biomedical Signal Processing and Control. 2010;5:45-52.

11. Rivadeneira PS, Moog CH. Impulsive control of singleinput nonlinear systems with application to HIV dynamics. Appl Math Comput. 2012;18:8462-8474.

12. Adams BM, Banks HT, Kwon HD, et al. Dynamic multidrug therapies for HIV: optimal and STI approaches. Math Biosci Eng. 2004;1:223-41.

13. Chang H, Astolfi A. Control of HIV infection dynamics. IEEE Control Systems. 2008;28:28-39.

14. Ahr B, Hebmann VR, Devaux C, et al. Apoptosis of uninfected cells induced by HIV envelope glycoproteins. Retrovirology. 2004;1:12-34.

15. Craig I, Xia X. Can HIV/AIDS be controlled? IEEE Control Systems. 2005;80-83.

16. Israel-Ballard K, Ziermann R, Leutenegger C, et al. Taqman RT-PCR and Versant HIV-1 RNA 3.0 (bDNA) assay quantification of HIV-1 RNA viral load in breast milk. J Clin Virol. 2005;34:253-256.

17. Delfraissy JF. Prise en charge des personnes infectées par le VIH : Recommandations du groupe d'experts. Flammarion, Médecine-Sciences: Paris; 2004.

18. U.S. Department of Health and Human Services. Guidelines for the use of antiretroviral agents in HIV-1-infected adults and adolescents, May 2010. Available at http://www.aidsinfo .nih.gov/guidelines

19. Badley AD. Cell Death During HIV Infection. CRC Press: Boca Raton, FL; 2005.

20. Gougeon ML, Montagnier L. Programmed cell death as a mechanism of CD4 and CD8 T cell depletion in AIDS: molecular control and effect of highly active anti-retroviral therapy. Ann NY Acad Sci. 1999;887:199-212.

21. Herbein G, Mahlknecht U, Batliwalla F, et al. Apoptosis of $\mathrm{CD} 8+\mathrm{T}$ cells is mediated by macrophages through interaction of HIV gp120 with chemokine receptor CXCR4. Nature. 1998;395:189-194.

22. Pantaleo G, Fauci AS. Apoptosis in HIV infection. Nat Med. 1995;1:118-120.

23. Vassena L, Proschan M, Fauci AS, et al. Interleukin 7 reduces the level of spontaneous apoptosis in CD4+ and CD8 + T cells from HIV-1 infected individuals. Proc Natl Acad Sci U S A. 2007;104:2355-2360.

24. Yue FY, Kovacs CM, Dimayuga RC, et al. Preferential apoptosis of HIV-1 specific CD4 + T cells. J Immunol. 2005; 174:2196-2204.

25. Berger A, Scherzed L, Stürmer M, et al. Comparative evaluation of the COBAS Amplicor HIV-1 Monitor ${ }^{\mathrm{TM}}$ Ultrasensitive Test, the new COBAS AmpliPrep/COBAS Amplicor HIV-1 Monitor $^{\mathrm{TM}}$ and the Versant HIV RNA 3.0 assays for quantitation of HIV-1 RNA in plasma samples. J Clin Virol. 2005;33:43-51.

26. Firme Roche. Fiches techniques, 2003. Available at http:// www.roche-diagnostics.fr

27. Galli R, Merrick L, Friesenhahn M, et al. Comprehensive comparison of the Versant HIV-1 RNA 3.0 (bDNA) and COBAS Amplicor HIV-1 Monitor 1.5 assays on 1000 clinical specimens. J Clin Virol. 2005;34:245-252.

28. Prud'homme IT, Kim JE, Pilon RG, et al. Amplicor HIV monitor, NASBA HIV-1 RNA QT and quantiplex HIV 
RNA version 2.0 viral load assays: a Canadian evaluation. J Clin Virol. 1998;11:189-202.

29. Rivadeneira PS, Moog CH, Stan GB, et al. Mathematical modeling of HIV dynamics after antiretroviral therapy initiation: a clinical research study. AIDS Res Hum Retroviruses. 2014;30:831-834.

30. Xia X, Moog CH. Identifiability of nonlinear systems with application to HIV/AIDS models. IEEE Transactions on Automatic Control. 2003;48:330-336.

31. Alvarez-Ramirez J, Meraz M, Velasco-Hernandez JX. Feedback control of the chemotherapy of HIV. Int J Bifurcation Chaos. 2000;10:2207-2219.

32. Costanza V, Rivadeneira PS, Biafore F, et al. A closed-loop approach to antiretroviral therapies for HIV infection. Biomedical Signal Processing and Control. 2009;4:139-148.

33. Jeffrey MA, Xia X, Craig I. When to initiate HIV therapy: a control theoretic approach. IEEE Trans Biomed Eng. 2003; 50:1213-1220.

34. Ouattara DA, Moog CH. Identification, linéarisation et commande optimale du modèle 3D de l'infection VIH-1. In Conférence Internationale Francophone d'Automatique, CIFA 2006, Bordeaux, France, May 2006.

35. Shim H, Han SJ, Chung CC, et al. Optimal scheduling of drug treatment for HIV infection: continuous dose control and receding horizon control. International Journal of Control, Automation, and Systems 2003;1:282-288.

36. Zurakowski R, Teel RA. A model predictive control based scheduling method for HIV therapy. J Theoret Biol. 2006; 238:368-382.

37. Hernandez-Vargas E, Colaneri P, Middleton R, et al. Discrete-time control for switched positive systems with application to mitigating viral escape. International Journal of Robust Nonlinear Control. 2011;21:93-111.

38. Legrand M, Comets E, Aymard G, et al. An in vivo pharmacokinetic/pharmacodynamic model for antiretroviral combination. HIV Clin Trials. 2003;4:170-183.

39. Mhawej MJ, Moog CH, Biafore F. The HIV dynamics is a single input system. In Proceedings of the 13th International Conference on Biomedical Engineering, Singapore, December 2008.

40. Nordic Medical Research Council's HIV Therapy Group. Double blind dose-response study of zidovudine in AIDS and advanced HIV infection. BMJ. 1992;304:13-17.
41. Siegel L, El-Sadr W. New perspectives in HIV treatment interruption: the SMART study. In: The PRN Notebook. Braun JF, Pozo MD (eds.) Physicians' Research Network: New York; vol. 11, pp. 8-9; 2006.

42. Hoffmann C, Rockstroh JK, Kamps BS (eds.). HIV Medicine 2007. Flying Publishers, 2007. Available at http:// hivmedicine.com/hivmedicine2007.pdf

43. Ramratnam BS, Bonhoeffer S, Binley J, et al. Rapid production and clearance of HIV-1 and hepatitis $\mathrm{C}$ virus assessed by large volume plasma apheresis. Lancet. 1999;354: 1782-1785.

44. Markowitz M, Louie M, Hurley A, et al. A novel antiviral intervention results in more accurate assessment of human immunodeficiency virus type 1 replication dynamics and T-cell decay in vivo. J Virol. 2003;77:5037-5038.

45. Luo R, Piovoso MJ, Martinez-Picado J, et al. HIV model parameter estimates from interruption trial data including drug efficacy and reservoir dynamics. PLoS ONE. 2012;7: e40198.

46. Putter H, Heisterkamp SH, Lange JMA, et al. A Bayesian approach to parameter estimation in HIV dynamical models. Stat Med. 2002;21:2199-2214.

47. Huang Y, Wu H, Acosta EP. Hierarchical Bayesian inference for HIV dynamic differential equation models incorporating multiple treatment factors. Biomed J. 2010;52: 470-486.

Address correspondence to: Dr. Pablo S. Rivadeneira Nonlinear System Group INTEC - CONICET Güemes 3450, C.P. 3000 Santa Fe Argentina

E-mail: psrivade@santafe-conicet.gov.ar

Abbreviation Used

$\mathrm{ZDV}=$ zidovudine 